\title{
Représentations sociales sur l'école et stratégies déployées par des parents récemment immigrés pour soutenir l'expérience socioscolaire de leurs enfants dans la société d'accueil : regards croisés de parents et d'intervenants
}

\author{
Josée Charette
}

Volume 37, numéro 1, été 2018

La recherche qualitative, un univers de nuances

URI : https://id.erudit.org/iderudit/1049458ar

DOI : https://doi.org/10.7202/1049458ar

\section{Aller au sommaire du numéro}

\section{Éditeur(s)}

Association pour la recherche qualitative (ARQ)

\section{ISSN}

1715-8702 (numérique)

\section{Découvrir la revue}

\section{Citer cet article}

Charette, J. (2018). Représentations sociales sur l'école et stratégies déployées par des parents récemment immigrés pour soutenir l'expérience socioscolaire de leurs enfants dans la société d'accueil : regards croisés de parents et d'intervenants. Recherches qualitatives, 37(1), 117-139.

https://doi.org/10.7202/1049458ar

\section{Résumé de l'article}

Cette recherche étudie les représentations sociales (RS) de parents d'élèves récemment immigrés au Québec sur les systèmes scolaires de leurs sociétés d'accueil et d'origine ainsi que les stratégies déployées par ces derniers pour soutenir l'expérience socioscolaire de leurs enfants en contexte migratoire. Selon une démarche qualitative interprétative exploratoire, la collecte de données a croisé le regard de 25 parents d'élèves québécois récemment immigrés et de six intervenants et intervenantes communautaires scolaires interculturels (ICSI) rencontrés dans le cadre d'entrevues semi-dirigées. La technique d'associations libres a aussi été mise à profit auprès des parents afin de favoriser l'accès à la complexité de leurs RS. Les résultats montrent l'ancrage des RS des parents dans leur expérience migratoire, comme dans les motifs d'immigration et dans les conditions d'établissement expérimentées dans la société d'accueil. Aussi, les résultats illustrent des RS structurées autour de quatre grands thèmes : l'intégration scolaire, sociale et professionnelle assurée par l'école; le développement intellectuel et la forme scolaire; divers enjeux de socialisation véhiculés à l'école et dans la société québécoise; les relations école-familles immigrantes. Au sujet des stratégies parentales déployées pour soutenir l'expérience socioscolaire des enfants, il semble que celles qui s'actualisent plus particulièrement dans l'intimité du milieu familial ou dans divers contextes de la communauté soient peu visibles pour le milieu scolaire, bien que nombreuses et souvent marquées par une proactivité importante. Le croisement des données recueillies auprès des parents et des ICSI permet aussi de préciser les besoins d'accompagnement de parents récemment immigrés pour une meilleure maîtrise des enjeux du milieu scolaire de la société d'accueil.
Tous droits réservés @ Association pour la recherche qualitative (ARQ), 2018
Ce document est protégé par la loi sur le droit d'auteur. L'utilisation des services d'Érudit (y compris la reproduction) est assujettie à sa politique d'utilisation que vous pouvez consulter en ligne.

https://apropos.erudit.org/fr/usagers/politique-dutilisation/ 


\title{
Texte lauréat du prix Jean-Marie-Van-der-Maren Concours 2017
}

\section{Représentations sociales sur l'école et stratégies déployées par des parents récemment immigrés pour soutenir l'expérience socioscolaire de leurs enfants dans la société d'accueil : regards croisés de parents et d'intervenants}

Josée Charette, Ph. D.

Université de Sherbrooke, Québec, Canada

\begin{abstract}
Résumé
Cette recherche étudie les représentations sociales (RS) de parents d'élèves récemment immigrés au Québec sur les systèmes scolaires de leurs sociétés d'accueil et d'origine ainsi que les stratégies déployées par ces derniers pour soutenir l'expérience socioscolaire de leurs enfants en contexte migratoire. Selon une démarche qualitative interprétative exploratoire, la collecte de données a croisé le regard de 25 parents d'élèves québécois récemment immigrés et de six intervenants et intervenantes communautaires scolaires interculturels (ICSI) rencontrés dans le cadre d'entrevues semi-dirigées. La technique d'associations libres a aussi été mise à profit auprès des parents afin de favoriser l'accès à la complexité de leurs RS. Les résultats montrent l'ancrage des RS des parents dans leur expérience migratoire, comme dans les motifs d'immigration et dans les conditions d'établissement expérimentées dans la société d'accueil. Aussi, les résultats illustrent des RS structurées autour de quatre grands thèmes : l'intégration scolaire, sociale et professionnelle assurée par l'école; le développement intellectuel et la forme scolaire; divers enjeux de socialisation véhiculés à l'école et dans la société québécoise; les relations école-familles immigrantes. Au sujet des stratégies parentales déployées pour soutenir l'expérience socioscolaire des enfants, il semble que celles qui s'actualisent plus particulièrement dans l'intimité du milieu familial ou dans divers contextes de la communauté soient peu visibles pour le milieu scolaire, bien que nombreuses et souvent marquées par une proactivité importante. Le croisement des données recueillies auprès des parents et des ICSI permet aussi de préciser les besoins d'accompagnement de parents récemment immigrés pour une meilleure maîtrise des enjeux du milieu scolaire de la société d'accueil.
\end{abstract}

RECHERCHES QUALITATIVES - Vol. 37(1), pp. 117-139.

LA RECHERCHE QUALITATIVE, UN UNIVERS DE NUANCES

ISSN 1715-8702 - http://www.recherche-qualitative.qc.ca/revue/

(C) 2018 Association pour la recherche qualitative 


\author{
Mots clés \\ REPRÉSENTATIONS SOCIALES (RS), PARENTS D'ÉLÈVES RÉCEMMENT IMMIGRÉS, SYSTÈMES \\ SCOLAIRES, STRATÉGIES PARENTALES DE SOUTIEN SCOLAIRE, INTERVENANT(E)S \\ COMMUNAUTAIRES SCOLAIRES INTERCULTURELS (ICSI), ENTREVUES SEMI-DIRIGÉES, TECHNIQUE \\ D’ASSOCIATIONS LIBRES
}

\title{
Introduction
}

$\mathrm{Au}$ cours des dernières années, le nombre d'élèves immigrants a augmenté dans les écoles du Québec, ces derniers représentaient 18,4\% de la population scolaire québécoise au cours de l'année scolaire 2007-2008 et 23,7\% au cours de l'année scolaire 2011-2012 (ministère de l'Éducation, du Loisir et du Sport [MELS], 2014a). Avec la diversification de la population scolaire, la recherche et le milieu scolaire se sont abondamment penchés sur les problématiques d'intégration et de persévérance scolaires au regard de diverses populations immigrantes (Audet, Potvin, Carignan, \& Bilodeau, 2010; Kanouté, Vatz Laaroussi, Rachédi, \& Tchimou Doffouchi, 2008; Mc Andrew et al., 2015; MELS, 2009). Comme lieu d'instruction, de qualification et de socialisation, l'école est effectivement interpellée à favoriser la réussite scolaire de tous les élèves québécois (MELS, 2009).

Pour la majorité des familles qui immigrent avec des enfants en âge de fréquenter l'école, la réussite scolaire de ces derniers dans la société d'accueil représente un enjeu capital du projet d'immigration (Bahi \& Piquemal, 2013; Changkakoti \& Akkari, 2008; Este \& Ngo, 2011; Kanouté \& Lafortune, 2010; Mondain \& Couton, 2011; Suárez-Orozco, Carhill, \& Chuang, 2011; Vatz Laaroussi, Rachédi, \& Helly, 2008). Ainsi, pour ces familles, la toile de fond constituée de défis généraux de l'immigration laisse clairement transparaître la problématique de l'exercice de la parentalité en situation d'acculturation et, en outre, de l'exercice du rôle de parent d'élève (Kanouté \& Lafortune, 2011a). Au Québec, dans une perspective de réussite éducative et scolaire, le rôle de parent d'élève se structure grandement autour de la participation parentale dans le parcours scolaire des enfants (MELS, 2009, 2014b). Désirant soutenir leurs enfants, mais ne détenant pas toutes les informations sur le milieu scolaire de la société d'accueil, plusieurs parents récemment immigrés fonderaient leur rôle de parent d'élève sur des représentations de l'école et de la scolarisation inspirées de leur pays d'origine (Robergeau, 2007; St-Fleur, 2007; Vatz Laaroussi, Tremblay, Corriveau, \& Duplain, 1999).

À la lumière de cette problématique, la question suivante est donc posée : en considérant la toile de fond des conditions générales de l'immigration et à travers les représentations sociales de parents récemment immigrés au Québec, de quelles manières ces derniers actualisent-ils leur rôle de parent d'élèves dans la société d'accueil québécoise? 


\section{Cadre conceptuel}

Le cadre conceptuel qui soutient le présent projet de recherche a été bâti à partir des trois thèmes suivants :

1. L'expérience socioscolaire des élèves récemment immigrés (Audet et al., 2010; Kanouté et al., 2008; Mc Andrew et al., 2015; Suárez-Orozco et al., 2011; Vatz Laaroussi, Rachédi, Kanouté, \& Duchesne, 2005): Il a été choisi de recourir au concept d'expérience socioscolaire dans le cadre du présent projet de recherche plutôt qu'à celui de réussite scolaire, par exemple, parce que le concept semble mieux décrire la complexité de l'expérience des élèves à l'école, en tenant compte de dimensions diverses (composantes scolaires, affectives, psychologiques, sociales, etc.). Aussi, le concept reconnaît l'apport de divers protagonistes et de leurs interactions entre eux comme soutien à l'expérience socioscolaire des élèves, s'ancrant dans une perspective écosystémique de la réussite scolaire. Aussi, il semble que ce concept recoupe les trois missions de l'école québécoise, soit de qualifier, d'instruire et de socialiser. Cette perspective de l'expérience socioscolaire a inspiré l'élaboration des schémas d'entrevues administrés aux parents et aux ICSI.

2. Le rapport à l'école et à la scolarisation de parents d'élèves récemment immigrés (Changkakoti \& Akkari, 2008; Epstein, 2011; Hohl, 1996; Jeynes, 2005; Vatz Laaroussi et al., 2005) : En se penchant sur le rapport à l'école de parents récemment immigrés, il paraît nécessaire de considérer le projet migratoire familial et divers enjeux d'établissement dans la société d'accueil comme toile de fond à la mobilisation scolaire. Aussi, il semble que plusieurs éléments concrets et implicites sont susceptibles de varier d'un système scolaire à l'autre, entraînant des ajustements divers pour les parents récemment immigrés. Les écrits explorés ont montré que le rôle de parent d'élève se décline sous des dimensions et dans des contextes divers. Pour les parents immigrants, il semble que la communauté prenne souvent une place importante dans les relations école-familles et que certaines déclinaisons du rôle de parent d'élève soient peu visibles pour le milieu scolaire. Ces constats ont guidé la présente étude vers des déclinaisons de l'implication parentale actualisées en marge de l'école et vers l'intérêt de rencontrer des protagonistes de la communauté dans le cadre de la collecte de données.

3. Le concept de représentation sociale [RS] (Doise, 1989; Garnier \& Rouquette, 2000; Jodelet, 2003; Moliner, 1996; Rateau, Moliner, Guimelli, \& Abric, 2012; Valence, 2010): Les RS se développent lorsqu'il est impossible pour une personne ou pour un groupe de rassembler l'intégralité des informations sur un objet social qui représente un enjeu. Comme il a été souligné précédemment, la réussite scolaire des enfants représente un enjeu majeur à l'effectivité de 
plusieurs projets migratoires familiaux. Ainsi, l'élaboration de RS sur le système scolaire de la société d'accueil permet aux parents de saisir la nature de cet objet social en le rattachant à des catégories familières, souvent liées au système scolaire de la société d'origine. Aussi, l'élaboration de RS sur le système scolaire de la société d'accueil constitue une façon pour les parents récemment immigrés de se construire un cadre commun de références et de prendre position dans les rapports sociaux avec les autres groupes de l'environnement social (les autres parents d'élèves, le personnel du milieu scolaire, les enfants, etc.). Ces constats ont inspiré la réflexion suivante: une meilleure connaissance des représentations des parents pourrait aider à décrypter leurs attitudes et leurs prises de position envers l'institution scolaire et divers enjeux associés, ce qui serait favorable pour l'établissement de relations école-familles efficaces et pour l'exercice du rôle parental et l'actualisation du rôle de parent d'élève en contexte migratoire.

Si des études se sont penchées sur des enjeux pédagogiques des relations écolefamilles immigrantes (Vatz Laaroussi, Kanouté, \& Rachédi, 2008), sur des enjeux linguistiques qui touchent les parents immigrants (De Koninck \& Armand, 2011), sur l'implication parentale en général (Deslandes \& Bertrand, 2004; Epstein, 2011; Fan \& Chen, 2001; Larivée, 2011) et sur l'implication parentale en contexte d'immigration (Jeynes, 2005; Moreno \& Chuang, 2011; Vatz Laaroussi et al., 2005; Vatz Laaroussi, Kanouté et al., 2008; Villiger, Wandeler, \& Niggli, 2014), aucune ne semble avoir étudié les RS qu'ont des parents récemment immigrés de l'école du pays d'origine et de la société d'accueil, tout en croisant leur regard avec le point de vue privilégié d'intervenants et intervenantes des milieux communautaire et scolaire. Ainsi, ce cadre conceptuel a mené à la formulation des objectifs de recherche suivants :

1. Décrire et analyser les RS que des parents d'élèves récemment immigrés ont des systèmes scolaires de leurs sociétés d'accueil et d'origine.

2. Décrire et analyser les stratégies déployées par des parents récemment immigrés pour soutenir l'expérience socioscolaire de leurs enfants dans la société d'accueil.

3. Décrire et analyser le regard porté par des ICSI sur le rapport à l'école et à la scolarisation de parents d'élèves récemment immigrés.

L'originalité de l'étude réside dans :

1) Le recours aux $R S$ pour étudier le rapport à l'école de parents récemment immigrés : la présente étude interroge les représentations des parents sur les systèmes scolaires de leurs sociétés d'accueil et d'origine ainsi que des enjeux qui y sont associés de façon plutôt exhaustive, ce que des recherches précédentes semblent avoir fait de façon plus succincte. L'angle des RS est également innovant pour étudier l'actualisation du rôle de parent d'élève en contexte 
migratoire puisqu'il prend en compte divers contextes sociaux qui sous-tendent cette actualisation.

2) La considération du contexte prémigratoire des parents : l'étude s'intéresse au rôle parental et au rôle de parent d'élève avec le parcours migratoire comme toile de fond.

3) Le fait de croiser deux points de vue sur la situation d'intérêt : l'étude croise le regard de parents récemment immigrés au Québec et d'ICSI, ce qu'aucune recherche ne semble avoir fait jusqu'à maintenant.

\section{Cadre méthodologique}

À l'instar d'autres écrits, nous estimons qu'il existe diverses manières d'interroger le monde (De Ketele \& Maroy, 2006; Pourtois, Desmets, \& Lahaye, 2006). En concordance avec les objectifs de la présente recherche, une démarche qualitative interprétative exploratoire semble la plus adéquate et la plus cohérente. La démarche qualitative exploratoire favorise l'accès à un ensemble « de données sur les perceptions de protagonistes locaux "de l'intérieur", à l'aide d'un processus d'attention approfondie, de compréhension empathique (Verstehen) et de préconceptions mises en suspens ou entre parenthèses sur les sujets abordés » (Miles \& Huberman, 2003, p. 21). Groulx (1997) soulève aussi l'apport des démarches qualitatives pour étudier « les spécificités socioculturelles de milieux de vie » (p. 58) en favorisant la mise en lumière de points de vue peu visibles, comme c'est parfois le cas des parents immigrants ou de protagonistes $\mathrm{du}$ milieu communautaire. Au regard du contexte migratoire en particulier, Kanouté et Lafortune (2011b) soulignent l'apport de la démarche qualitative pour faire ressortir à la fois le pluralisme et la singularité des processus d'acculturation.

\section{Recrutement de l'échantillon}

Puisque ce projet de recherche s'inscrit dans une démarche qualitative interprétative exploratoire, l'échantillonnage de type non probabiliste orienté a été privilégié. Celuici donne effectivement la possibilité d'accéder à une connaissance détaillée de personnes ancrées dans un contexte social selon des critères spécifiques (Miles \& Huberman, 2003; Mongeau, 2008; Poupart et al., 1997). L'échantillon a par la suite été complété en utilisant la technique «boule de neige» (Mongeau, 2008; Pires, 1997). Des critères de sélection ont été fixés dans un plan de recrutement et ont été légèrement révisés au contact du terrain. Ainsi, il avait été planifié de rencontrer des familles immigrantes :

- Demeurant au Québec depuis six mois à cinq ans : Le délai de six mois visait à laisser aux familles un temps d'adaptation à leur nouvel environnement avant que ces dernières ne soient sollicitées pour la participation à une recherche. Le délai de cinq ans était fixé selon une définition de Statistique Canada qui 
considère les familles récemment immigrées comme étant « établies au Canada depuis moins de cinq ans » (2008, Encadré 1). À la suite de la rencontre de diverses familles, il a été décidé d'élargir le critère de sélection jusqu'à dix ans de séjour au Québec. Cette décision a été prise en raison du terrain : il a en effet été constaté que certains défis avec l'école, notamment, persistent pour plusieurs familles, même après un séjour de plus de cinq ans au Québec.

- S'exprimant en français ou en anglais : Alors qu'il était prévu de recruter des parents en mesure de s'exprimer en français ou en anglais, l'espagnol a finalement aussi été mis à profit (troisième langue maîtrisée par la chercheuse), ce choix étant l'occasion d'enrichir les données recueillies et de documenter le rapport à l'école de parents d'élèves qui ne maîtrisent ni le français ni l'anglais.

- Ayant des enfants qui fréquentent l'école publique francophone: Ce critère sur la fréquentation de l'école publique a été levé puisque des familles dont les enfants fréquentaient des écoles francophones privées ont signalé leur intérêt pour la collecte de données. Ainsi, des stratégies inédites ont été révélées pour soutenir l'expérience socioscolaire des enfants en contexte migratoire, dont le financement de l'école privée, malgré de modestes moyens financiers.

Ainsi, en tenant compte des contraintes du terrain de recherche, les parents recrutés pour la collecte de données répondaient finalement aux critères suivants :

- Ils sont des parents d'élèves immigrants.

- Ils vivent au Québec depuis six mois à dix ans.

- Ils proviennent d'au moins quatre pays d'origine différents.

- Ils résident dans la grande région métropolitaine de Montréal.

- Ils parlent le français, l'anglais ou l'espagnol.

- Leurs enfants fréquentent l'école publique ou privée francophone au Québec.

- Au moins un de leurs enfants est né au pays d'origine.

Pour favoriser un regard croisé sur la situation d'intérêt, un deuxième groupe de sujets a été formé, il était prévu que celui-ci soit composé d'au moins six ICSI, hommes et femmes, certains nés au Québec et d'autres à l'extérieur du Canada, travaillant ${ }^{1}$ dans au moins trois régions administratives différentes au Québec. En fin de compte, la flexibilité dont nous avons fait preuve dans le recrutement des participants et des participantes pour la collecte de données est courante dans les recherches qualitatives (Blanchet \& Gotman, 2013). Elle aura permis d'enrichir l'échantillon ainsi que l'analyse des données. 


\section{Stratégies d'échantillonnage}

À la suite de l'acceptation du devis par le jury et de la délivrance du certificat d'éthique par l'Université de Montréal, des stratégies de recrutement ont été mises en place. Dans plusieurs terrains de recherche, les «modes d'accès indirects » aux participants et participantes, " par l'entremise de tiers, institutionnels ou personnels », pour «maximiser les chances d'acceptation» (Blanchet \& Gotman, 2013, p. 53) sont mis à profit. Dans cette logique, il a été choisi de passer par l'intermédiaire de quatre organismes communautaires et d'une enseignante du primaire pour recruter l'échantillon souhaité, ces intermédiaires ont été choisis pour leur emplacement géographique sur l'île de Montréal et pour leurs missions d'accueil et d'intégration de populations récemment immigrées. Ainsi, des collaborations ont été établies avec divers protagonistes qui nous ont recommandé des sujets aux profils recherchés. Lorsque nous avions en mains les coordonnées de ces personnes, nous communiquions avec elles pour leur présenter le projet de recherche dans la langue de leur choix, sonder leur intérêt à y participer et prendre rendez-vous pour une rencontre en personne.

De façon générale, les familles et les ICSI contactés se sont montrés très ouverts dès le premier contact téléphonique. La relation de confiance établie entre les sujets et les intermédiaires des milieux communautaires et scolaires nous a probablement été favorable, puisque lors d'un entretien, «la personne interviewée tend à se faire une représentation de l'interviewer relative à celle qu'il a de la personne ayant joué le rôle d'intermédiaire pour la prise de contact » (Blanchet, 2003, p. 150).

\section{Échantillon}

Ainsi, un échantillon de recherche a été rassemblé, composé de 17 mères, 3 pères et 5 couples père-mère, vivant à Montréal depuis 6 mois à 9 ans, ayant au moins un enfant né au pays d'origine et dont les enfants fréquentent une école francophone publique ou privée au Québec. Les parents rencontrés étaient originaires de 11 pays d'origine différents (Algérie, Cameroun, Cuba, Égypte, Haïti, Maroc, Mexique, Moldavie, Pérou, Rwanda et Tunisie) et avaient 7 langues maternelles variées (arabe, créole, espagnol, kabyle, kinyarwanda, medumba et roumain).

Six ICSI, aux caractéristiques suivantes ont aussi été rencontrés: quatre femmes et deux hommes, œuvrant dans quatre régions administratives du Québec: les Laurentides (1), Laval (1), Montréal (3) et la Capitale-Nationale (1), nés au Canada (2/6), au Mexique (2/6), en Algérie (1/6) et en Colombie (1/6). Les langues maternelles des ICSI sont l'arabe (1/6), l'espagnol (3/6) et le français $(2 / 6)$. Au moment des entrevues, l'expérience de travail des ICSI dans la profession allait d'un peu plus d'un mois à huit ans. 


\section{Méthodes de collecte de données : entrevues semi-dirigées et technique d'associations libres}

La démarche de collecte de données s'est faite à l'aide de deux méthodes. D'abord, pour les deux groupes de sujets, l'entrevue semi-dirigée a été employée. L'emploi de cette méthode a été privilégié puisqu'elle permet aux sujets de parcourir des questions ouvertes comme ils l'entendent, selon un axe central proposé par la chercheuse (Mucchielli, 2009). L'entrevue semi-dirigée enrichit le matériel d'analyse et le contenu d'une recherche en favorisant l'afflux d'informations nouvelles n'ayant pas émergé du cadre conceptuel (Poupart, 1997). Également, l'entrevue recueille le point de vue de protagonistes sur un sujet en particulier, en accédant de manière compréhensive à leurs représentations et à leurs perceptions, en obtenant un matériel ancré dans leurs réalités personnelles et sociales (Roussiau \& Bonardi, 2001; Van der Maren, 1996), ce qui concorde avec les objectifs de la présente recherche relatifs aux parents ainsi qu'aux ICSI.

Les schémas d'entrevue étaient constitués des sections suivantes :

a) Pour les parents : projet migratoire en général et expérience comme parent; représentations des systèmes scolaires des sociétés d'accueil et d'origine et expérience comme parent d'élève; perception du parent de l'expérience socioscolaire de l'enfant dans la société d'accueil et stratégies parentales déployées pour soutenir cette expérience.

b) Pour les ICSI : nature du travail d'ICSI; perception des besoins des familles immigrantes relativement à l'école; perception des stratégies déployées par les parents pour soutenir l'expérience socioscolaire de leurs enfants; pratiques déployées par les ICSI pour faciliter le rapport à l'école des parents récemment immigrés.

L'entrevue est une méthode de collecte de données qui comporte toutefois certaines contraintes. Pour les parents plus précisément, il a été nécessaire de faire preuve de vigilance au regard de la possible émergence de barrières linguistiques, étant donné que le français n'était que rarement leur langue maternelle. Pour diminuer l'impact de cette limite, il leur a été proposé de choisir la langue employée dans le cadre de l'entrevue, parmi le français, l'anglais ou l'espagnol.

La méthode d'entrevue peut aussi induire le phénomène de désirabilité sociale, selon lequel les personnes interrogées tentent de répondre aux questions pour préserver leur estime de soi, autant à leurs propres yeux qu'aux yeux des autres (Lamoureux, 2000). Pour contrer cet effet, la technique de substitution a été employée dans l'élaboration de certaines questions d'entrevue (Abric, 2003). Cette dernière vise à réduire la pression normative chez les personnes rencontrées en leur permettant d'exprimer leurs idées en les attribuant à une catégorie d'appartenance plus large, par 
exemple: selon vous, est-ce que l'école est compréhensive envers les parents immigrants? Cette technique invite par contre à la prudence puisque les éléments ressortis par les sujets n'appartiennent qu'hypothétiquement à leurs prises de position sur un objet. En effet, les sujets peuvent aussi «produire la représentation du groupe de référence qu'on leur propose » (Abric, 2003, p. 78) sans nécessairement y adhérer.

Aussi, afin de contrer le phénomène de désirabilité sociale et de favoriser l'accès à la complexité des RS des parents (Abric, 1994; Apostolidis, 2003; Grenon, Larose, \& Carignan, 2013), l'entrevue semi-dirigée a été combinée à la technique d'associations libres. Celle-ci a servi de prémisse à l'entrevue semi-dirigée des parents et visait surtout à favoriser leur spontanéité ainsi que la réduction de certains biais liés à l'entrevue. Concrètement, une feuille blanche a été présentée aux parents au milieu de laquelle était inscrite l'expression école au Québec, school in Quebec ou escuela en Quebec. La consigne suivante leur était donnée (traduite en anglais ou en espagnol le cas échéant) : «Écrivez ou dictez-moi tous les mots qui vous viennent à l'esprit quand vous voyez l'expression école au Québec ». Aucune contrainte de temps ou de nombre de mots ne leur était imposée. Il a été choisi d'administrer la technique d'associations libres de deux façons; les parents interrogés pouvaient choisir de compléter l'exercice à l'oral, auquel cas l'intervieweuse notait pour eux les mots dictés sur la feuille, ou à l'écrit, par eux-mêmes. Cette précaution a été prise afin d'éviter d'augmenter le sentiment de vulnérabilité sociale ressentie chez certaines personnes interrogées.

La technique d'associations libres a ainsi permis aux parents d'exprimer librement et spontanément leurs représentations sur les systèmes scolaires de leurs sociétés d'accueil et d'origine (Carassus \& Dosquet, 2010; Vidal, Rateau, \& Moliner, 2006), sans les soucis lexicaux et syntaxiques qu'exige l'entrevue. Les divers éléments amenés par les parents durant l'exercice d'associations libres ont ensuite été repris au courant de l'entrevue, après avoir établi un lien de confiance avec eux.

La technique d'associations libres comporte toutefois aussi certaines limites. Entre autres, cette méthode de collecte de données peut induire certaines barrières linguistiques pour les parents qui complètent parfois l'exercice dans une langue qui n'est pas leur langue maternelle. Afin de diminuer les impacts de cette limite, il a été proposé aux parents de compléter l'exercice en anglais, en français ou en espagnol. Aussi, les éléments qui ressortent de la technique d'associations libres doivent être analysés et interprétés en fonction du contexte de production (Carassus \& Dosquet, 2010; Vergès, 1992). Selon Valence (2010), les représentations contextualisées où il y a objectivation des connaissances ne devraient être qu'une première étape à l'étude des RS. "Autrement dit, quand l'analyse a délimité l'organisation d'un champ représentationnel, elle doit ensuite appréhender sa dimension socio-objective » (Valence, 2010, p. 71). Ainsi, l'emploi de l'entrevue semi-dirigée permettait 
d'atteindre la dimension sociale des représentations et de l'insérer dans des dynamiques d'immigration et de réussite scolaire.

\section{Traitement et analyse des données}

En concordance avec les objectifs de cette recherche, l'analyse des données a été qualitative. Ainsi, les discours des parents émis au sujet de l'exercice d'associations libres et les entrevues ont été transcrits sous forme de verbatim dans le logiciel Word, puis ont ensuite été importés dans le logiciel d'analyse qualitative QDA Miner. Ce logiciel, développé par Provalis Research, permet de «coder des données textuelles, d'annoter, d'extraire et de réviser des données et des documents codés » (Recherches Provalis, n.d., p. 4$)^{2}$.

Le traitement des données s'est fait selon une analyse thématique de contenu. Pour Mucchielli (2009), l'analyse de contenu désigne « un ensemble de méthodes d'analyse de documents, le plus souvent textuels, permettant d'expliciter le ou les sens qui y sont contenus ou les manières dont ils parviennent à faire effet de sens » (p. 36). Elle s'est donc exercée sur un corpus regroupant un ensemble de verbatim d'entrevues, travail étant destiné à "expliciter in fine la ou les significations » des données recueillies (Mucchielli, 2009, p. 36). Selon Blanchet et Gotman (2013), l'analyse de contenu ne doit pas avoir une prétention d'objectivité absolue, car elle est une activité de production de sens, donc une lecture orientée d'une certaine façon.

Plus précisément, le codage consiste à donner des étiquettes à des unités de sens repérés dans des verbatim, les unités de sens étant souvent des extraits formés de mots, de phrases ou même de paragraphes (Miles \& Huberman, 2003). Tout en restant le plus près du sens commun, les codes sont une façon simple de transformer des données brutes en une première formulation signifiante (Mucchielli, 2009). Dans le cadre de la présente étude, le codage a été mixte, c'est-à-dire que nous l'avons d'abord construit à partir d'une liste de codes inspirés du cadre conceptuel élaboré et des schémas d'entrevue. Les thèmes étaient associés à des catégories et à des sous-catégories, permettant de «subsumer un sens plus général sous un ensemble d'éléments bruts du corpus ou d'éléments déjà traités et dénommés (codifiés) » (Mucchielli, 2009, p. 17), lesquels étaient ensuite associés à des codes. Dans le cas des parents, par exemple, ce travail a mené au codage suivant :

- Représentations des parents au sujet du système scolaire québécois (thème) forme scolaire (catégorie) - méthodes d'enseignement (sous-catégorie), regroupement représenté sous le code $R S-F O R-M E T H$;

- Stratégies pour soutenir l'expérience socioscolaire des enfants (thème) - maison (catégorie) - aide dans les devoirs (sous-catégorie), l'ensemble étant représenté sous le code STRA-MAIS-DEV. 
Ensuite, des thèmes généraux récurrents et des catégories qui n'avaient pas été déjà soulignés dans la liste initiale des codes ont été repérés dans le corpus (Mucchielli, 2009). Pour ce faire, une lecture intensive du corpus a permis de s'approprier le matériel recueilli lors de la collecte de données et de faire ressortir, dans chacune des entrevues, les unités de sens à coder et éventuellement à analyser; il s'agissait de faire ressortir l'information du bruit (Van der Maren, 1996). Cette lecture des entrevues a aussi fait émerger de nouveaux thèmes qui ont mené à la création de nouveaux codes et à l'enrichissement de la liste initiale de codes. Aussi, quelques codes déjà existants ont été modifiés dans leur formulation afin de mieux tenir compte du vocabulaire utilisé spontanément par les personnes interrogées (Van der Maren, 1996). Voici quelques codes qui ont émergé du corpus :

- Représentations des parents au sujet du système scolaire québécois (thème) mission de qualification (catégorie) - enseignement de l'anglais (souscatégorie), le tout représenté sous le code $R S-Q U A L-A N G$;

- Stratégies pour soutenir l'expérience socioscolaire des enfants (thème) - maison (catégorie) - choix de l'école privée (sous-catégorie), regroupement représenté sous le code STRA-MAIS-ÉCOPRI.

Afin d'obtenir un codage efficace et rigoureux, il a été vérifié qu'un même extrait n'était pas codé par une autre unité de sens concurrente et que les mêmes codes étaient attribués de manière systématique aux mêmes unités de sens (Van der Maren, 1996). Pour ce faire, un lexique définissant les codes accompagnés d'exemples a été construit dans le logiciel QDA Miner, évitant de cette façon le plus possible des interprétations multiples d'un même code (Van der Maren, 1996).

À la toute fin du travail de codage, une deuxième vérification de tous les codes a été effectuée pour s'assurer que ces derniers étaient «exclusifs, afin d'éviter les chevauchements, exhaustifs, afin de couvrir la totalité du document, évidents, afin d'assurer une certaine objectivité, et pertinents par rapport aux objectifs de recherche » (Dorais, 1993, p. 15).

Selon Mucchielli (2009), la catégorisation des données représente « un début de théorisation, car le chercheur fait appel à l'ensemble de ses connaissances, [...] la catégorisation met en œuvre des paradigmes de ses références qui interviennent alors comme système implicite de perception-compréhension » (p. 17). Lors de cette étude, l'analyse des données et le travail d'interprétation se sont d'abord faits de façon verticale, c'est-à-dire que les thèmes abordés par chacun des sujets ont été passés en revue séparément pour en faire la synthèse (Blanchet \& Gotman, 2013). Puis, une analyse transversale a permis de révéler les différentes formes sous lesquelles apparaissait le même thème d'un sujet à l'autre (Blanchet \& Gotman, 2013). L'analyse transversale des données visait principalement à repérer les répétitions, les contradictions, les compléments d'information, les divergences et les ressemblances 
entre les sujets de notre étude, comme c'est particulièrement le propos d'une démarche qualitative de recherche (Paillé \& Mucchielli, 2012).

Afin de bien saisir la portée des données recueillies inhérentes à une recherche, il est nécessaire de reconnaitre les forces et les faiblesses de la méthodologie retenue. Bien que l'approche qualitative présente plusieurs forces au regard de la présente étude, cette dernière invite toutefois à la prudence en ce qui concerne notre subjectivité en tant que chercheuse dans l'entreprise de la démarche scientifique, où nous représentons le principal outil de collecte de données (Anadón, 2013; Karsenti \& Savoie-Zajc, 2004; Lamoureux, 2000). Afin de contrer cette limite, nous avons tenté de demeurer objective en nous distanciant de nos présupposés, de nos idéologies, de nos postulats et de nos autres a priori, et ce, à toutes les étapes du processus de recherche (Lessard-Hébert, Goyette, \& Boutin, 1995; Van der Maren, 2009). Aussi, s'il est vrai que le cadre conceptuel a inspiré la construction des schémas d'entrevue, la forme semi-dirigée des entretiens a favorisé l'afflux d'informations nouvelles (Poupart, 1997). Selon Crahay (2006), « l'enfermement dans la singularité de l'individu » (p. 36) est une autre limite de l'approche qualitative; l'analyse transversale des données a tenté d'en diminuer l'impact.

Concernant le traitement et l'analyse des données, des précautions ont été prises afin d'éviter que celles-ci soient tributaires de nos interprétations (Carassus \& Dosquet, 2010; Vidal et al., 2006). La traduction des données invitait aussi à la vigilance. Dans ce cas-ci, il a été vérifié que la traduction des outils de collecte de données et des données recueillies était la plus exacte possible et que le sens des propos tenus par les sujets était maintenu lors des traductions. Pour ce faire, une personne bilingue a relu les verbatim d'entrevues en espagnol et en anglais et a confirmé les résumés d'entrevues faits en français.

Cela dit, que révèlent les résultats sur le rapport à l'école et à la scolarisation de parents d'élèves récemment immigrés au Québec?

\section{Résultats, discussion et contribution à l'avancement des connaissances}

Comme il avait été anticipé, les RS des parents sur l'école du Québec sont, entre autres, inspirées du système scolaire connu dans leur pays d'origine (Robergeau, 2007; St-Fleur, 2007; Vatz Laaroussi et al., 1999). Par contre, les résultats de la présente recherche illustrent surtout des RS tournées vers l'avenir, qui interpellent principalement la capacité de l'école à intégrer leurs enfants dans les milieux scolaire et socioprofessionnel de la société d'accueil. Ainsi, les résultats mettent en exergue l'association étroite que plusieurs parents font entre l'école et sa mission de qualification; ils font précisément ressortir l'ancrage des RS des parents récemment immigrés sur le système scolaire québécois dans leur expérience migratoire. Entre autres, il semble que les motifs d'immigration de plusieurs familles mènent les parents à se positionner sur divers aspects qui concernent l'école et la scolarisation dans la 
société d'accueil, par exemple : les modalités de la persévérance scolaire ou le niveau d'efficacité d'enseignement de certaines disciplines comme les mathématiques et l'anglais. L'étude soutient donc la nécessité que les protagonistes du milieu scolaire connaissent et considèrent certains enjeux liés au parcours migratoire des familles pour mieux comprendre diverses prises de position des parents envers l'école et envers la scolarisation de leurs enfants.

À l'instar d'autres travaux, les résultats de la présente recherche soulignent aussi la place stratégique du milieu scolaire dans l'intégration de nombreuses familles récemment immigrées dans la société d'accueil (Audet et al., 2010; Kanouté \& Lafortune, 2011a; Mc Andrew et al., 2015). Ils montrent surtout la place de l'école en tant que territoire de négociation de certaines valeurs de socialisation véhiculées dans la société québécoise : définition du rôle parental, rapport des enfants aux adultes, droit des enfants et développement de l'autonomie. Ainsi, l'étude attire l'attention sur le rôle incontournable de l'école dans le processus d'acculturation qui touche précisément le rôle parental et, en outre, celui de parent d'élève. Les résultats soulignent aussi l'intérêt de considérer des malentendus susceptibles de survenir entre l'école et la famille au sujet de certains enjeux de socialisation et l'intérêt d'en discuter avec les parents avant même que ces derniers troublent les relations école-familles. D'ailleurs, les ICSI semblent être des ressources stratégiques pour faciliter le processus d'acculturation des parents récemment immigrés au Québec et pour favoriser leur intégration dans le milieu scolaire québécois, en soutenant la communication/médiation interculturelle entre l'école et les familles.

Aussi, comme d'autres travaux le soulignent (Bahi \& Piquemal, 2013; Vatz Laaroussi et al., 2005), les résultats de la présente recherche soulèvent la place centrale de la réussite scolaire des enfants dans l'effectivité de nombreux projets migratoires familiaux. Par ailleurs, ils font davantage ressortir l'importance accordée par les parents à une expérience généralement positive des enfants à l'école du Québec. En effet, plusieurs parents de la présente étude semblent tout aussi préoccupés, sinon plus, par l'intégration sociale, le développement global et l'actualisation du potentiel de leurs enfants à l'école québécoise, que par l'obtention de bons résultats scolaires. Dans le même sens, les données recueillies montrent l'impossibilité d'occulter le rôle de certains membres du personnel scolaire, à leur connaissance ou non, comme vecteur de résilience du projet migratoire familial, comme le soulignent aussi Vatz Laaroussi et al. (2005). Par conséquent, l'étude soutient l'idée selon laquelle l'école et ses protagonistes participent à la qualité de l'expérience migratoire des familles avec des enfants en âge de fréquenter l'école. Ainsi, les résultats mettent en évidence la nécessité de former les membres du personnel scolaire aux enjeux de l'immigration et de les sensibiliser à la place stratégique qu'ils détiennent dans les processus d'accueil et d'intégration à l'école de nombreuses familles nouvellement arrivées dans la province. À cet effet, les ICSI semblent posséder une expertise non négligeable des 
enjeux généraux de l'immigration en famille et des connaissances sur les familles ancrées dans des réalités locales en particulier, leur permettant ainsi d'assurer des formations pertinentes auprès du personnel scolaire.

Dans un autre ordre d'idées, l'étude tend à montrer la contribution du capital social des parents récemment immigrés dans la construction de leurs RS sur l'école et dans l'élaboration de stratégies de soutien scolaire pour leurs enfants. En effet, il semble que l'investissement des parents dans divers réseaux de sociabilité (pairs immigrants, organismes communautaires, réseaux étudiants par un retour aux études) favorise la construction de représentations plus complexes sur l'école et, par conséquent, les parents prennent position de façon plus stratégique au regard du parcours scolaire de leurs enfants. Le réseautage amènerait les parents à poser un regard plus large sur le milieu scolaire, plutôt que sur la seule école fréquentée par leurs enfants, et ils seraient ainsi plus en mesure de prendre des décisions qui favorisent l'actualisation du capital humain de leurs enfants. Ce projet de recherche attire l'attention sur l'importance des réseaux informels qui soutiennent les parents dans leur intégration à l'école et dans la société d'accueil plus largement. Il soulève la pertinence de soutenir le développement du capital social familial à travers les ressources mises à la disposition des familles par les milieux scolaires et communautaires. Ainsi, il semble pertinent d'accorder une plus grande place aux réseaux de sociabilité informels ainsi qu'aux espaces de médiation qui permettent d'améliorer l'expérience scolaire des familles.

L'analyse de l'implication parentale dans le suivi scolaire montre un ensemble de stratégies mobilisées par les familles rencontrées, dont plusieurs sont corroborées par le témoignage des ICSI. La stratégies déployées par les parents ont été classées en trois catégories, soit celles qui se déploient à la maison, à l'école et en lien avec la communauté. Les résultats mettent en lumière un décalage intéressant entre la mobilisation et la proactivité accrues de nombreux parents et l'invisibilité de ces dernières pour le milieu scolaire, surtout lorsque la mobilisation parentale est actualisée dans l'intimité du milieu familial ou dans divers contextes de la communauté. Aussi, de nombreuses stratégies parentales qui visent l'actualisation maximale du capital humain des enfants se déclinent à l'écart de l'école. Ainsi, plusieurs parents se mobilisent pour permettre à leurs enfants d'accéder aux meilleures conditions de scolarisation existantes dans la société d'accueil : préparer les enfants à l'entrée à l'école québécoise en leur payant des cours particuliers de français, faire des exercices scolaires supplémentaires avec eux, choisir l'école des enfants en dehors de celle assignée par la commission scolaire, opter pour l'école privée malgré des moyens financiers restreints, etc. La présente étude s'inscrit dans la même lignée que celles de Jeynes (2005) et Vatz Laaroussi et al. (2005) qui soulignent la forte mobilisation de certains parents dans le parcours scolaire de leurs enfants, malgré peu de contacts directs avec l'école, parfois. 
L'étude a aussi permis de constater que certaines stratégies déployées par les parents ne sont pas toujours vues positivement par le milieu scolaire. Il semble d'ailleurs que l'actualisation du rôle de parent d'élève " à l'école » dépende en partie des possibilités que le personnel scolaire donne aux parents de déroger aux modalités attendues par l'école, une contrainte à laquelle les parents ont beaucoup moins à se soumettre dans le contexte de la maison ou de la communauté. Ainsi, les résultats de la présente recherche remettent en question l'aisance et la facilité du milieu scolaire à reconnaître des stratégies parentales de soutien scolaire qui diffèrent un peu de la définition du rôle de parent d'élève instituée par l'école ou qui se déploient à l'écart de l'école. Cette étude souligne donc la nécessité que le milieu scolaire connaisse et reconnaisse des déclinaisons du rôle de parent d'élève différentes de celles qui sont mises de l'avant par le milieu scolaire pour pouvoir valoriser d'autres façons « d'être parent d'élève ». Pour ce faire, il est nécessaire que les protagonistes du milieu scolaire se décentrent en vue d'admettre diverses perspectives. À cet égard, les ICSI semblent représenter une porte d'entrée intéressante pour l'école sur «la face cachée » de l'actualisation du rôle de parent d'élève.

Le croisement des regards des deux groupes de protagonistes a mis en évidence des besoins des parents d'élèves récemment immigrés ainsi que des pratiques d'ICSI qui semblent soutenir l'accompagnement de ces parents à l'école du Québec. À partir de cela, des pistes d'intervention qui se résument ainsi ont été élaborées :

- Permettre aux parents de partir du bon pied en mettant en œuvre un protocole d'accueil et d'intégration qui accorde explicitement une place aux parents d'élèves récemment immigrés.

- Donner accès aux parents récemment immigrés à une personne-ressource pour répondre à leurs questions sur l'école au fil de leur découverte du milieu scolaire.

- Contourner/diminuer certaines contraintes structurelles rencontrées par les familles récemment immigrées qui peuvent influencer leur rapport à l'école en leur proposant des solutions concrètes à divers de leurs besoins.

- Reconnaître diverses postures aux parents d'élèves récemment immigrés.

- Favoriser l'établissement de collaborations école-famille-communauté où la part de chacun des protagonistes est bien définie.

En somme, en plus d'avoir atteint les objectifs de recherche ci-haut mentionnés, la présente étude a fait en sorte de :

- Mieux connaître et de considérer divers enjeux de l'immigration en famille (situation prémigratoire, contexte scolaire du pays d'origine, conditions d'établissement dans la société d'accueil); informer les protagonistes des milieux scolaires et communautaires sur les RS de parents récemment immigrés 
des systèmes scolaires de leurs sociétés d'accueil et d'origine; permettre aux protagonistes de prendre connaissance de certaines prises de position des parents; favoriser la mise en place de formation pour les protagonistes du milieu scolaire sur les dynamiques et les enjeux de l'immigration; sensibiliser le milieu scolaire à sa place stratégique dans les processus d'acculturation, d'accueil, d'intégration et de résilience de plusieurs familles récemment immigrées.

- Documenter des déclinaisons du rôle de parent d'élève peu ressorties dans les recherches et peu visibles pour le milieu scolaire; documenter les besoins d'accompagnement de parents d'élèves récemment immigrés dans le milieu scolaire québécois pour favoriser l'actualisation de leur rôle de parent d'élève; mettre en lumière diverses déclinaisons du rôle de parent d'élève qui ne s'inscrivent pas exactement dans les modalités de l'implication parentale proposées par l'école ou qui s'actualisent en dehors du milieu scolaire; permettre au milieu scolaire de valoriser diverses déclinaisons du rôle de parent d'élève et permettre aux parents de se sentir reconnus dans leurs façons de faire; soutenir les parents récemment immigrés dans l'actualisation de leur rôle de parent d'élève; reconnaître l'apport des parents dans l'intégration des enfants à l'école du Québec.

- Documenter le travail fait par les ICSI auprès des familles et auprès des écoles; considérer les ICSI dans les processus d'accueil et d'intégration de familles nouvellement arrivées à l'école du Québec; donner la parole à des protagonistes qui ont une place stratégique dans les processus d'accueil et d'intégration des familles immigrantes à l'école du Québec, mais dont le discours est peu exploité par la recherche; capter l'expérience des familles récemment immigrées à l'école du Québec selon le regard des ICSI; accéder à un point de vue significatif sur l'expérience des familles récemment immigrées à l'école québécoise, ce qui semble avoir été moins étudié jusqu'à maintenant.

- Proposer des stratégies pour de meilleures conditions dans les relations école-famille-communauté; permettre au milieu scolaire de connaître et de reconnaître les espaces de médiation pour soutenir l'intégration des familles à l'école et l'expérience socioscolaire des jeunes; permettre au milieu scolaire de connaître et de reconnaître «la ressource ICSI »; proposer des stratégies pour une meilleure communication entre les divers protagonistes qui soutiennent l'expérience socioscolaire des élèves.

- Proposer des pistes pour mieux accompagner les parents d'élèves récemment immigrés à l'école de la société d'accueil; favoriser une expérience socioscolaire positive chez les élèves récemment immigrés au Québec en soutenant l'actualisation du rôle de parent d'élève. 
Bien que les résultats de la présente étude ne puissent pas être généralisés à tous les contextes, cette recherche aura servi, somme toute, à mieux comprendre l'expérience de parents d'élèves montréalais récemment immigrés dans le milieu scolaire québécois, à mieux saisir l'actualisation de leur rôle de parent d'élève dans le contexte d'immigration et à faire ressortir des pistes d'intervention qui permettent d'accompagner efficacement les parents d'élèves récemment immigrés. Ainsi, nous nous rangeons du côté d'autres écrits (Farmer \& Labrie, 2008; MELS, 2009) qui soulignent que l'école ne peut arriver à elle seule à soutenir l'intégration des familles récemment immigrées et la réussite scolaire des élèves récemment immigrés à l'école de la société d'accueil.

\section{Notes}

${ }^{1}$ Puisque les ICSI ne proviennent pas de ces régions administratives, mais y travaillent.

${ }^{2}$ www.provalisresearch.com/Documents/QDAMiner3f.pdf

\section{Références}

Abric, J.-C. (1994). Méthodologie de recueil des représentations sociales. Dans J.-C. Abric (Éd.), Pratiques sociales et représentations (pp. 59-82). Paris : Presses universitaires de France.

Abric, J.-C. (2003). Méthodes d'étude des représentations sociales. Saint-Agne : Érès.

Anadón, M. (2013). La recherche sociale et l'engagement du chercheur qualitatif : Défis du présent. Recherches qualitatives, Hors-série, 14, 5-14.

Apostolidis, T. (2003). Représentations sociales et triangulation: Enjeux théoricométhodologique. Dans J.-C. Abric (Éd.), Méthodes d'étude des représentations sociales (pp. 13-35). Saint-Agne : Érès.

Audet, G., Potvin, M., Carignan, N., \& Bilodeau, A. (2010). Famille et expérience socioscolaire d'élèves d'origine immigrée en contexte de défavorisation socioéconomique à Montréal. La parole aux jeunes. Dans G. Pronovost (Éd.), Familles et réussite éducative. Actes du symposium québécois de recherche sur la famille (pp. 55-71). Québec : Presses de 1'Université du Québec.

Bahi, B., \& Piquemal, N. (2013). Dépossession socio-économique, linguistique et résilience : Horizons de mobilité sociale chez les élèves immigrants, réfugiés au Manitoba. Cahiers franco-canadiens de l'Ouest, 25(1-2), 109-128.

Blanchet, A. (2003). Dire et faire dire : L'entretien. Paris : Armand Colin. 
Blanchet, A., \& Gotman, A. (2013). L'enquête et ses méthodes. L'entretien. Paris : Armand Colin.

Carassus, D., \& Dosquet, F. (2010, Novembre). Comment mieux connaître les membres de sa communauté virtuelle pour un parti politique. L'utilisation du concept de représentations sociales. Revue de littérature des méthodologies applicables. Communication présentée à la $15^{\mathrm{e}}$ IBIMA Conference, Caire, Égypte.

Changkakoti, N., \& Akkari, A. (2008). Familles et écoles dans un monde de diversité : Au-delà des malentendus. Revue des sciences de l'éducation, 34(2), 419-441.

Crahay, M. (2006). Qualitatif-quantitatif : Des enjeux méthodologiques convergents? Dans L. Paquay, M. Crahay, \& J.-M. De Ketele (Éds), L'analyse qualitative en éducation. Des pratiques de recherche aux critères de qualité (pp. 33-52). Bruxelles : De Boeck et Larcier.

De Ketele, J.-M., \& Maroy, C. (2006). Quels critères de qualité pour les recherches en éducation? Dans L. Paquay, M. Crahay, \& J.-M. De Ketele (Éds), L'analyse qualitative en éducation. Des pratiques de recherche aux critères de qualité (pp. 219-249). Bruxelles : De Boeck et Larcier.

De Koninck, Z., \& Armand, F. (2011, Hiver). Le choix des modèles de service offerts aux élèves issus de l'immigration au Québec: Entre réalisme, tradition et innovation. Canadian Issues, 29-34.

Deslandes, R., \& Bertrand, R. (2004). Motivation des parents à participer au suivi scolaire de leur enfant au primaire. Revue des sciences de l'éducation, 30(2), 411433.

Doise, W. (1989). Attitudes et représentations sociales. Dans D. Jodelet (Éd.), Les représentations sociales (pp. 220-237). Paris : Presses universitaires de France.

Dorais, M. (1993). Diversité et créativité en recherche qualitative. Service social, $42(2), 7-27$.

Epstein, J. L. (2011). School, family, and community partnerships: Preparing educators and improving schools. Boulder, CO : Westview.

Este, D., \& Ngo, H. V. (2011). A resilience framework to examine immigrant and refugee children and youth in Canada. Dans S. S. Chuang, \& R. P. Moreno (Éds), Immigrant children change, adaptation, and cultural transformation (pp. 27-49). Lanham, MD: Lexington books.

Fan, X., \& Chen, M. (2001). Parental involvement and students' academic achievement : A meta-analysis. Educational Psychology Review, 13(1), 1-22. 
Farmer, D., \& Labrie, N. (2008). Immigration et francophonie dans les écoles ontariennes : Comment se structurent les rapports entre les institutions, les parents et le monde communautaire? Revue des sciences de l'éducation, 34(2), 377-398.

Garnier, C., \& Rouquette, M.-L. (2000). Représentations sociales et éducation. Montréal : Nouvelles AMS.

Grenon, V., Larose, F., \& Carignan, I. (2013). Réflexions méthodologiques sur l'étude des représentations sociales : Rétrospectives de recherches antérieures. Phronesis, 2(2-3), 43-49.

Groulx, L.-H. (1997). Contribution de la recherche qualitative à la recherche sociale. Dans M. Lessard-Hébert, G. Goyette, \& G. Boutin (Éds), La recherche qualitative: Fondements et pratiques (pp. 55-82). Bruxelles: De Boeck Université.

Hohl, J. (1996). Qui sont «les parents»? Le rapport de parents immigrants analphabètes à l'école. Lien social et politiques, 35, 51-62. doi : 10.7202/005126ar

Jeynes, W. H. (2005). A meta-analysis of the relation of parental involvement to urban elementary school student academic achievement. Urban Education, 40(3), 237269.

Jodelet, D. (2003). Représentations sociales: Un domaine en expansion. Dans D. Jodelet (Éd.), Les représentations sociales (pp. 47-78). Paris : Presses universitaires de France.

Kanouté, F., \& Lafortune, G. (2010). Les familles immigrantes : mobilisation autour du projet scolaire des enfants. Nos diverses cités, 7, 143-150.

Kanouté, F., \& Lafortune, G. (2011a). Familles québécoises d'origine immigrantes. Les dynamiques de l'établissement. Montréal: Les Presses de l'Université de Montréal.

Kanouté, F., \& Lafortune, G. (2011b). La réussite scolaire des élèves d'origine immigrée : Réflexions sur quelques enjeux à Montréal. Éducation et francophonie, 39(1), 80-92.

Kanouté, F., Vatz Laaroussi, M., Rachédi, L., \& Tchimou Doffouchi, M. (2008). Familles et réussite scolaire d'élèves immigrants du secondaire. Revue des sciences de l'éducation. 34(2), 265-289.

Karsenti, T., \& Savoie-Zajc, L. (2004). La recherche en éducation: Étapes et approches. Sherbrooke : CRP.

Lamoureux, A. (2000). Recherche en méthodologie en sciences humaines. Québec: Études Vivantes. 
Larivée, S. J. (2011). Regards croisés sur l'implication parentale et les performances scolaires. Service social, 57(2), 5-19.

Lessard-Hébert, M., Goyette, G., \& Boutin, G. (1995). La recherche qualitative : Fondements et pratiques. Montréal : Éditions Nouvelles.

Mc Andrew, M., Balde, A., Bakhshaei, M., Tardif-Grenier, K., Audet, G., Armand, F., ... Rousseau, C. (Éds). (2015). La réussite éducative des élèves issus de l'immigration: Dix ans de recherche et d'intervention au Québec. Montréal : Les Presses de l'Université de Montréal.

Miles, M. B., \& Huberman, A. M. (2003) Analyse des données qualitatives. Bruxelles : De Boeck et Larcier.

Ministère de l'Éducation, du Loisir et du Sport (MELS). (2009). Tous ensemble pour la réussite scolaire. Québec: Gouvernement du Québec. Repéré à http://www.education.gouv.qc.ca/fileadmin/site_web/documents/dpse/formation_je unes/LEcoleJyTiens_TousEnsemblePourLaReussiteScolaire.pdf

Ministère de l'Éducation, du Loisir et du Sport (MELS). (2014a). Portrait statistique 2011-2012 des élèves issus de l'immigration. Formation générale des jeunes Édition 2013. Québec : Gouvernement du Québec.

Ministère de l'Éducation, du Loisir et du Sport (MELS). (2014b). Cadre de référence. Accueil et intégration des élèves issus de l'immigration au Québec. Québec: Gouvernement du Québec. Repéré à http://www.education.gouv.qc.ca/references/p ublications/resultats-de-la-recherche/detail/article/accueil-et-integration-deseleves-issus-de-limmigration-au-quebec-cadre-de-reference/

Moliner, P. (1996). Images et représentations sociales. Grenoble: Presses universitaires de Grenoble.

Mondain, N., \& Couton, P. (2011). L'insertion socio-éducative des enfants d'immigrants africains à Montréal et à Ottawa-Gatineau. Dans F. Kanouté, \& G. Lafortune (Éds), Familles québécoises d'origine immigrantes. Les dynamiques de l'établissement (pp. 59-71). Montréal : Les Presses de l'Université de Montréal.

Mongeau, P. (2008). Réaliser son mémoire ou sa thèse : Côté jeans et côté tenue de soirée. Québec : Presses de l'Université du Québec.

Moreno, R. P., \& Chuang, S. S. (2011). Challenges facing immigrant parents and their involvement in their children's schooling. Dans S. S. Chuang, \& R. P. Moreno (Éds), Immigrant children change, adaptation, and cultural transformation (pp. 239-254). Lanham, MD: Lexington books.

Mucchielli, A. (2009) Dictionnaire des méthodes qualitatives en sciences humaines et sociales. Paris : Armand Colin. 
Paillé, P., \& Mucchielli, A. (2012). L'analyse qualitative en sciences humaines et sociales. Paris : Armand Colin.

Pires, A. (1997). Échantillonnage et recherche qualitative: Essai théorique et méthodologique. Repéré à http://classiques.uqac.ca/contemporains/pires_alvaro/ec hantillonnage_recherche_qualitative/echantillon_recherche_qual.pdf

Poupart, J. (1997). L'entretien de type qualitatif: Considérations épistémologiques, théoriques et méthodologiques. Dans J. Poupart, L.-H. Groulx, J.-P. Deslauriers, A. Laperrière, R. Mayer, \& A. P. Pires (Éds), La recherche qualitative. Enjeux épistémologiques et méthodologiques (pp. 173-209). Boucherville : Gaëtan Morin.

Poupart, J., Groulx, L.-H., Deslauriers, J.-P., Laperrière, A., Mayer, R., \& Pires, A. P. (Éds). (1997). La recherche qualitative. Enjeux épistémologiques et méthodologiques. Boucherville : Gaëtan Morin.

Pourtois, J.-P., Desmets, H., \& Lahaye, W. (2006). Postures et démarches épistémiques en recherche. Dans P. Paillé (Éd.), La méthodologie qualitative. Postures de recherche et travail de terrain (pp. 169-200). Paris : Armand Colin.

Rateau, P., Moliner, P., Guimelli, C., \& Abric, J.-C. (2012). Social representation theory. Dans P. A. M. Van Lange, A. W. Kruglanski, \& E. T. Higgins (Éds), Handbook of theories of social psychology (pp. 477-497). Londres : Sage.

Recherches Provalis (n.d.). QDA Miner. Logiciel d'analyse qualitative des données [manuel de l'utilisateur]. Repéré à www.provalisresearch.com/Documents/QDAMi ner3f.pdf

Robergeau, D. (2007). Regards croisés sur la trajectoire scolaire d'élèves d'origine haïtienne en situation de réussite scolaire (Mémoire de maîtrise inédit). Université de Montréal, QC.

Roussiau, N., \& Bonardi, C. (2001). Les représentations sociales : État des lieux et perspectives. Sprimont : Mardaga.

Statistique Canada. (2008). Portrait des immigrants au Canada. Repéré à http://www.statcan.gc.ca/pub/85f0033m/2008018/findings-resultats/portraitfra.htm

St-Fleur, J. M. (2007). Le contexte prémigratoire et l'implication parentale des parents d'origine haïtienne dans le suivi scolaire de leurs enfants au Québec (Mémoire de maîtrise inédit). Université de Montréal, QC.

Suárez-Orozco, C., Carhill, A., \& Chuang, S. S. (2011). Immigrant children. Making a new life. Dans S. S. Chuang, \& R. P. Moreno (Éds), Immigrant children change, adaptation, and cultural transformation (pp. 7-26). Lanham, MD: Lexington books. 
Valence, A. (2010). Les représentations sociales. Bruxelles : De Boeck Université.

Van der Maren, J. M. (1996). Méthodes de recherche pour l'éducation (2 éd.). Montréal : Presses de l'Université de Montréal.

Van der Maren, J. M. (2009, Juin). La recherche qualitative, instrument stratégique d'émergence d'une discipline "éducation». Communication présentée au deuxième Colloque international francophone sur les méthodes qualitatives, Lille, France.

Vatz Laaroussi, M., Kanouté, F., \& Rachédi, L. (2008). Les divers modèles de collaboration famille immigrantes-écoles : De l'implication assignée au partenariat. Revue des sciences de l'éducation, 34(2), 291-311.

Vatz Laaroussi, M., Rachédi, L., \& Helly, D. (2008). Des ruptures migratoires à la transmission familiale : Un espace de production sociale. Dans M. Vatz Laaroussi, C. Bolzman, \& M. Lahlou (Éds), Familles migrantes au gré des ruptures. Tisser la transmission (pp. 16-35). Lyon : L'interdisciplinaire.

Vatz Laaroussi, M., Rachédi, L., Kanouté, F., \& Duchesne, K. (2005). Favoriser les collaborations familles immigrantes-écoles. Soutenir la réussite scolaire. Guide d'accompagnement. Sherbrooke : Université de Sherbrooke.

Vatz Laaroussi, M., Tremblay, P.-A., Corriveau, L., \& Duplain, M. (1999). Les histoires familiales au cœur des stratégies d'insertion: Trajectoires de migration en Estrie et au Saguenay-Lac-St-Jean. Rapport de recherche adressé au CQRS, Université de Sherbrooke.

Vergès, P. (1992). L'évocation de l'argent : Une méthode pour la définition du noyau central d'une représentation. Bulletin de psychologie, 45(406), 203-209.

Vidal, J., Rateau, P., \& Moliner, P. (2006). Les représentations en psychologie sociale. Dans N. Blanc (Éd.), Le concept de représentation en psychologie (pp. 11-42). Paris : In-Press Editions.

Villiger. C., Wandeler, C., \& Niggli, A. (2014). Explaining differences in reading motivation between immigrant and native students: The role of parental involvement. International Journal of Educational Research, 64, 12-25.

Josée Charrette mène actuellement une recherche postdoctorale à l'Université de Sherbrooke, à la Faculté d'éducation. Elle est aussi chargée de cours à l'Université du Québec à Montréal et à l'Université de Montréal, ainsi que collaboratrice à la Direction des services d'accueil et d'éducation interculturelle au ministère de l'Éducation. Ses travaux de recherche portent principalement sur les enjeux de la pluriethnicité en contextes éducatifs, des relations école- 
familles immigrantes et de l'expérience socioscolaire des élèves récemment immigrés. Ses travaux sont principalement ancrés dans des démarches qualitatives de recherche, privilégiant souvent des méthodes associatives pour favoriser la spontanéité des sujets rencontrés.

Pour joindre l'auteure : joseecharette1306@gmail.com 